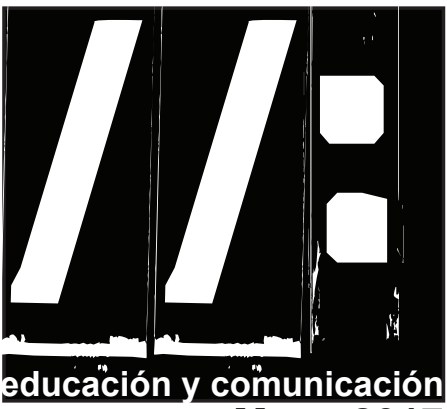

14:115-122 Mayo 2017

\section{LA ESCUELA FRENTE A LA GENERACIÓN MILLENNIALS}

\section{The school front generation millennials}

\author{
Dra. Teresa Montes de Oca Sánchez \\ Universidad Interamericana para el Desarrollo \\ Tlalnepantla. (México) \\ E.mail: tms_10@hotmail.com
}

Resumen:

En la actualidad se identifican estrategias educativas vinculadas al uso de la tecnología para la comunicación. Sin embrago, hoy en día, se tienen estudiantes universitarios y docentes de la llamada generación millennials, que han tenido influencia de estos medios por lo que están hiperconectados, al mismo tiempo que se reconocen capaces de realizar lo que se propongan. Estas características hacen necesaria el desarrollo de estrategias incluyentes que rescaten el uso de la tecnología que es de gran interés para estos alumnos pero, al mismo tiempo, no se olviden de aquellos que no cuentan con el manejo de dispositivos y pueden ser considerados como analfabetas digitales. Por otro lado, es importante reconocer los retos a los que se enfrentan los docentes que no utilizan estos medios por lo que existe una brecha digital que limita la comunicación en las aulas; por lo cual se requiere de docentes comprometidos, incluyentes, respetuosos y dispuestos a aprender unos de otros.

Palabras clave: Escuela del siglo XXI, generación millennials, profesorado, educación.

\title{
Abstract:
}

At present, educational strategies linked to the use of technology for communication are identified; however, there are currently university students and teachers of the so-called millennials generation; that have had influence of these means by which they are hyper connected, at the same time that they recognize themselves capable to realize what they are proposed. These characteristics make it necessary to include inclusive strategies that rescue the use of technology that is of great interest to these students, but at the same time do not forget those who do not have the device management and can be considered as digital illiterate. On the other hand, it is important to recognize the challenges faced by teachers who do not use these means, so there is a digital divide that limits communication in classrooms; For which it is necessary of collegiate committed, inclusive, respectful and willing to learn from each other

Key Words: School of the 21st century, generation millennials, professor, education.

Recibido: 21-01-2017 Revisado: 11-02-2017 Aceptado: 26-02-2017 Publicado: 01-05-2017 


\section{II: Educación, Comunicación y Cultura Contemporánea}

\section{Rasgos característicos de los millennials}

$\mathrm{M}$ ucho se ha discutido acerca de cómo aprenden los alumnos y las mejores estrategias para que accedan al conocimiento académico. Sin lugar a dudas, se reconoce la importancia de la formación docente que se mueva en múltiples áreas del conocimiento y que éstas se articulen de manera que el estudiante pueda darle sentido a los contenidos y, con ello, se consoliden los saberes necesarios para los profesionistas en formación. También se ha analizado en los últimos 10 años, cómo se han transformado las maneras de interacción en el aula y, por consecuencia, las necesidades de aprendizaje de los alumnos. Con frecuencia se hace mención sobre lo diferente que son los niños y jóvenes que hoy cursan la educación básica, sin embargo, ¿se reconoce la diferencia generacional que se observa en la educación media superior y superior?

Es por ello que en el sistema educativo mexicano, así como en muchas otras partes del mundo, se analizan las características de esta generación llamada Millennials o $\mathrm{Y}$, que tiene dentro de los rasgos que los identifican la facilidad para el uso de dispositivos electrónicos. Ellos tienen el privilegio de haber nacido con la revolución tecnológica. Por esta razón, utilizan formas diferentes de establecer canales de comunicación a las que se utilizaban por la generación X. Mientras que es, justamente, el acelerado desarrollo tecnológico el que da lugar a nuevas formas para acceder a la información por lo tanto las estrategias no pueden ser las mismas.

«Los nacidos entre 1980 y 2000, son los conocidos como Generación Y o millennial... Los millennials constituyen una generación con señas de identidad propias. Se ven a sí mismos como emprendedores que piensan en los demás. Su patrón de confianza es distinto al de las generaciones que le han precedido... Para esta generación, los medios sociales son más que una forma de comunicar, son la plataforma por la que dan sentido a su mundo. (Pintado 2014: 179)»

Es está la generación más incorporada al uso de Internet y los dispositivos electrónicos que los vinculan con sus pares, por lo que son altamente confiados con quiénes sienten empatía y con quiénes se observan en semejanzas en intereses y aficiones. Tienen, prácticamente, resuelto todo en la palma de la mano por lo que, también, se puede referir a ellos como nativos digitales, se mueven con gran destreza y resuelven situaciones en torno a la tecnología, por lo que suele decirse que tienen una lógica de computadora, lo que los hacen vertiginosos y fácilmente adaptables a los cambios en los sistemas operativos que revolucionan velozmente.

Estos millenials se han incorporado mediante el juego al uso de estos celulares, tablets y computadoras, ya que muchos padres de la generación $\mathrm{X}$ les entretuvieron con ellos, así como también representó un signo de estatus de la familia cuando los niños tenían juegos electrónicos portátiles, con lo que se acostumbraron a ellos y los integraron, definitivamente, a su entretenimiento. Suelen parecer jóvenes ensimismados, solitarios e individualistas. Sin embargo, se conectan muchas horas del día con amigos e intercambian información relevante para ellos, misma que no necesariamente sea valiosa para sus padres o maestros. Los millennials son chicos que prefieren el uso de redes sociales por encima de los convencionales e.mails, ya que la comunicación en grupos y veloz les proporciona la inmediatez que requieren, mueven los dedos 


\section{1/: La escuela frente a la generación millennials}

ágilmente en el teclado miniatura y desesperan cuando no reciben respuesta en breve. Tienen el privilegio de poder hacer multiplicidad de tareas en un espacio físico muy pequeño, su habitación, desde este sitio son capaces de indagar, analizar e incluso crear. Para ellos la búsqueda de información se remite a una gran cantidad de resultados arrojados en fracciones de segundo, por lo que son capaces de manipular también múltiples ventanas al mismo tiempo, están expuestos a una hiper estimulación visual y auditiva.

Se habla mucho sobre la falta de compromiso, de visión y de esfuerzo de la generación millennials. Se les plantean como jóvenes desinteresados por los demás, que solo buscan satisfacer sus necesidades personales y sus gustos más superfluos, se habla de una cifra muy alta de ellos que no trabajan, no estudian y no tienen proyectos a mediano o largo plazo. Igualmente, viven con sus padres o son ellos quienes ayudan a pagar el alquiler de la vivienda. Pero, también, se tienen en esta generación a quienes han creado empresas sólidas que han incrementado sus ventas, es decir, se encuentran dentro de la generación Y. Son personas que se vinculan con otros, tienen compromisos con proyectos suyos o en donde participan, son quienes generan ideas y las exponen con seguridad. "Los millenials traen consigo una nueva generación de emprendedores, que gracias a una creatividad explosiva están redefiniendo la economía, la sociedad, la comunicación humana y generado nuevos modelos de negocios, rompiendo paradigmas en muy diversos ámbitos" (González, 2015: 79).

Gracias a las oportunidades de múltiples pantallas portátiles en donde se accede a Internet, sus funciones también se han multiplicado. Hoy en día se puede hacer la compra en línea, cerrar un boleto de avión, acceder a la cafetería y obtener beneficios al utilizar las aplicaciones. Lo que ayuda a que los dispositivos sean una fuente importante en sus vidas y que las actividades que realizan de forma cotidiana por esta generación sea más frecuente, por ejemplo, acceder a redes sociales, blogs y plataformas, que leer los periódicos convencionales. Es decir, buscan información en fuentes diferentes a las de la generación $X$, que confía más en expertos que en los pares. $\mathrm{Y}$ es aquí donde empiezan a visualizarse las diferencias sutiles pero, evidentemente, importantes en las formas de comunicar de acuerdo a la generación a la que se pertenezca.

\section{Oportunidades educativas con los jóvenes millennials.}

Para los alumnos millennials, hay una serie de contenidos educativos alejados de su centro de interés y de las necesidades de aprendizaje para resolver situaciones cotidianas, por lo que se distraen con facilidad, se cansan y se muestran desinteresados por lo que los docentes pretenden comunicar. Por lo tanto, se inicia unos procesos que van por caminos diferentes y al no coincidir se tienen muchos problemas para comunicar en las aulas. Hacen uso de manera frecuente de los dispositivos portátiles que son más comunes y accesibles para la población general. Estos condicionantes han replanteado algunos de los contenidos educativos, sin que se modifiquen o integren las tecnologías como medio de exploración académica de forma innovadora. Ya que, en ciertos casos, los dispositivos multimedia se utilizan de la misma manera que el tradicional libro de texto. En este sentido, los docentes lo usan para que los estudiantes lean y resuman la información, por lo que no se logra motivar a la realización 


\section{II: Educación, Comunicación y Cultura Contemporánea}

de las tareas escolares, al contrario, se genera mayor resistencia.

Una de las más evidentes barreras para articular el aprendizaje de las tecnologías de la información y la comunicación es la brecha existente entre la hiper conectividad de los chicos y el analfabetismo cibernético de los docentes; cuando al tratar de utilizar estas herramientas titubean ante la inseguridad que les representa la lógica de los recursos. Por lo que algunos prefieren no hacer uso de ellos, y es que...

«Enseñar y aprender en las situaciones creadas por las nuevas tecnologías exige flexibilidad, espacio y tiempo, personalmente y en grupo, menos contenidos fijos y procesos abiertos de investigación y comunicación. La construcción de conocimiento, a partir de las tecnologías impregna a las personas de una mayor motivación e interés hacia el aprendizaje, además de facilitar la interacción entre el alumno-profesor y profesor-estudiante, así como del alumnado y los docentes entre sí. Asimismo, posibilita el desarrollo de la autonomía en el proceso de aprendizaje y su autorregulación. (Vázquez, 2011: 17-18)»

Es, precisamente, esta flexibilidad la que no es parte de las generaciones anteriores a la generación Y, en donde las personas tienen una probabilidad de comunicación gracias a las pantallas portátiles. Por esta razón, a partir de una incorporación forzada en donde los contenidos educativos se alejan de la oportunidad que dan los recursos tecnológicos, son de tal forma, tan artificiales, que no corresponden a la necesidad formativa e informativa de los chicos millennials. Es evidente, y mucho se ha hablado al respecto, que una comunicación efectiva con éstos jóvenes implica el uso de los recursos que ellos dominan y por lo tanto les interesan. No obstante, como se ha mencionado anteriormente, para los profesores es un gran reto, que solamente se puede solventar al establecer los adecuados puentes de comunicación entre los diferentes grupos de edad que se encuentran en las escuelas.

Por lo tanto las escuelas, docentes y en general la infraestructura tecnológica deberán dar soporte suficiente para satisfacer las necesidades de conexión y la movilidad de datos en las plataformas que puedan ofrecer de manera casi permanente el uso de recursos en grandes cantidades de personas conectadas al mismo tiempo. Reconocer determinadas limitaciones en ciertas regiones específicas permite establecer las opciones que se puedan realizar de manera real y no dejarse llevar por falsas expectativas, lo que puede suceder con entusiasmo desbordado. Es requisito indispensable considerar los diferentes contextos educativos para poder dar respuestas adecuadas a la formación de los millennials. De lo contrario, se corren riesgos de sobre tecnologizar los planes y programas sin que ello se pueda corresponder a las necesidades e intereses de formación de los estudiantes.

Se puede observar el gran impacto que tiene la tecnología en la vida cotidiana de los jóvenes; sin lugar a dudas no es lo único que les interesa, les gusta, les funciona, sobre todo por el tipo de comunidad de la que se trate. Lo que no exime, de una u otra forma, del manejo y necesidades para incorporarse al siglo XXI, en donde la interconexión es cada vez más frecuente como necesaria. Se encuentra que:

«(...) existe otra forma de manifestación de la brecha digital relacionada con las posibilidades de acceso a la tecnología por parte de grupos sociales desfavorecidos. Son varios los millones de personas que hoy no tienen la posibilidad de acceder a servicios de Internet u otros sistemas digitalizados de información en red, y mucho menos a la posesión de estas tecnologías en su hogar. (Barrios, 2009: 271)» 


\section{I/: La escuela frente a la generación millennials}

De tal forma que es necesario eliminar el analfabetismo cibernético, aportar a la educación una posibilidad para la comunicación y la información al alcance de todos, democratizar el uso de dispositivos para acceder a las redes y los contenidos que ahí se encuentran. Una vez que se tenga la cobertura se podrá realizar esta digitalización en el uso de recursos educativos, en donde se puedan movilizar aprendizajes académicos y para la vida. Para algunos de los estudiantes nativos digitales, ésta podrá ser una actividad natural. Sin embargo, para quienes no lo sea se deberá iniciar y mantener para su aprovechamiento permanente. Ya que el docente no deberá olvidarse de aquellos estudiantes que no se encuentran familiarizados con el uso tecnológico.

En México, la educación de estos jóvenes millennials se ofrece en la educación pública o privada, lo que distancia a unos chicos de otros. Para que esto no impacte de manera negativa en su formación, la escuela deberá proveer los recursos que apoyen a impulsar el uso de la tecnología, ya que algunas instituciones llegan a tener computadoras insuficientes o en mal estado. Pudiéndose tener redes que no dan soporte a la cantidad de dispositivos móviles o fijos, con lo que implementarla en las sesiones resultaría frustrante y limitada.

En realidad, el incremento del uso de esta tecnología permite establecer diferentes formas de acceder a la información y, evidentemente, facilitan la comunicación entre las personas. Pero a qué se debe que los millennials se articulen con tanta facilidad y pasen tantas horas en las redes sociales. Se puede pensar en procesos de aprendizaje distintos a los que se tenían en otras generaciones. Sin lugar a dudas, el contexto tiene un peso importante, pero lo más relevante puede ser el reconocimiento de las inteligencias múltiples donde Howard Gardner (2011) identifica las diferencias individuales en los procesos de adquisición de saberes, desde los canales por los cuales cada uno se implica para el saber. Se habla de estudiantes que son dispersos, aislados, poco interesados; lo que sucede es que los chicos se encuentran inmersos en una amplia estimulación visual y auditiva, de la que recuperan y manipulan información. El pensamiento de estos estudiantes suele ser igual de vertiginoso que el ritmo de vida que llevan.

\section{Retos para los docentes de los millennials}

Evidentemente, uno de los retos más obvio tiene que ver con el manejo de los medios tecnológicos. Esta brecha puede ser una barrera de comunicación y para el aprendizaje que los docentes se estén planteando para con sus estudiantes. Sin embargo, no sería lo único, ya que este manejo puede tener ciertas trampas, así como los padres, los profesores no pueden pretender asociarse en las redes como amigos de ellos. Los riesgos que tiene la facilidad en la comunicación van desde las falsas creencias acerca de la amistad vinculada a la calificación escolar, hasta acusaciones sobre relaciones interpersonales más cercanas de lo que debería ser.

Los millennials presentan agilidad mental y una fuerte carga de autoestima y de mensajes en los que se les ha dicho que son capaces de realizar cualquier cosa que se propongan, lo les da seguridad y fortaleza para enfrentar diversas situaciones. Ellos han aprendido a poner en duda, prácticamente, todo lo que se les plantea. Son jóvenes que tienen a su alcance una multitud de resultados de una búsqueda en un tiempo, extremada- 


\section{II: Educación, Comunicación y Cultura Contemporánea}

mente, corto. Se cuentan, actualmente, con buscadores en los que ellos confían ampliamente, de manera que sus consultas no son directamente con maestros, son en Internet, por lo que este recurso es indispensable en su actuar cotidiano. Gracias a la inmediatez se les puede encontrar ansiosos en el aula, al encontrarse con un espacio que poco se ha adaptado a este siglo XXI. Sobre todo con profesores que dicten cátedra en lugar de involucrarlos en proyectos que los pongan en acción física y mental.

De tal forma que si se requiere de su atención, se deben enviar mensajes visuales y auditivos, videos e imágenes que puedan ser de interés para ellos. Pero, sobre todo, promover la realización de éstos en donde se pongan en juego la elaboración experimental de estos medios. Es, en este sentido, donde la equidad juega un papel muy importante. Por lo que los docentes recurren a estrategias diversas para acercar a los alumnos de manera que ninguno se quede rezagado. Sin embargo, se puede argumentar la falta de interés o de disciplina para las actividades escolares, y se evidencia la falta de vinculación de los que aprenden con los que enseñan, y viceversa. Los canales de comunicación y la actitud para acercarse al conocimiento deben estar presentes, de lo contrario, no existe movilización en el saber.

«El desarrollo de competencias para cumplir con la demanda de la aldea global del siglo XXI el docente es parte de una educación de vanguardia. Existe la necesidad actual de que éste no solo cuente con conocimientos académicos de su cátedra en particular sino que posea habilidades académicas, administrativas y humano-sociales requeridas para hacer una diferencia competitiva. (Aldape, 2008: 26)»

Como consecuencia de la alta y diversa especialización requerida para la función docente, un reto más hace referencia a la pérdida de la motivación para el ejercicio de la profesión. Y, con ello, para la cobertura en las escuelas de profesionales que estén comprometidos, conozcan y sean sensibles a la individualización en la enseñanza, al reconocer en los estudiantes las habilidades y destrezas que puedan ser la plataforma para su formación profesionalizante. La baja demanda en las escuelas formadoras puede implicar un peligro a corto plazo que limite a los docentes en ejercicio.

Los chicos generación millennials, en la actualidad, tienen ya responsabilidades laborales. Por lo tanto, también algunos de ellos se encuentran en la docencia. La convivencia con ellos podría ser abrumadora sino se tiene la habilidad para auto reconocerse en las fortalezas aún sin pertenecer a esta generación. El dominio que tienen acerca del uso de la tecnología, así como la amplia gama de saberes y un pensamiento divergente pareciera ser su carta de presentación con la que ganarían espacios laborales. En el caso particular de la docencia, se encuentran además de éstos factores, elementos igualmente valiosos, como la experiencia y el manejo de grupos, además de la alta especialización en la que generaciones anteriores han sido formadas; que también son factor de posibilidades de éxito. De tal manera que no es un riesgo que se encuentren distintas generaciones conviviendo laboralmente. Incluso, al contrario, esta es la ganancia que es necesario trabajar en la formación para, realmente, obtener los beneficios que implica esta condición.

\section{Conclusiones}

La generación millennials, es una oportunidad para incorporar nuevos planteamientos y formas de ver la función pedagógica, en donde lo más importante 


\section{I/: La escuela frente a la generación millennials}

sea la persona en el centro de la acción educativa. El profesional del siglo XXI, tiene características que le ayudan a identificarse plenamente, es un chico multifacético que vive plenamente el aquí y el ahora, suele ser inquieto y su proceso de atención pareciera ser superficial. Sin embargo, los asuntos de su interés le vinculan fuertemente en la tarea para el logro de sus propósitos. Evidentemente, en las aulas universitarias existen jóvenes con estos rasgos. Son ellos los que pasan largas horas frente a las pantallas, los que prefieren canales de información que se articulen con lo visual, auditivo o cinestésico. Son aquellos que pierden el interés al no encontrar una aplicación inmediata.

Para ello, se han planteado las características que tienen los chicos de la generación millennials, también conocidos como generación $\mathrm{Y}$, o nativos digitales. Con estas categorías se han reconocido a aquellos quienes dominan los recursos tecnológicos. Lamentablemente, no se puede hablar de una democratización de esta tecnología aplicada a la comunicación y a la educación. En la actualidad, se cuentan con poblaciones que no acceden a los mismos, ya sea por no contar con la infraestructura en la zona para la conexión a Internet o por la dificultad para acceder a los dispositivos por ser muy caros. De esta manera, se deberá tener precaución al incorporar las mismas para la enseñanza superior. Las estrategias que se utilicen deberán contemplar la alfabetización para algunos y la cobertura para los alumnos que no cuenten con computadoras, tablets o celulares con capacidad para el manejo de datos y plataformas educativas.

Con los millennials en las aulas ya sea como alumnos o como docentes, se requieren procesos en los que tanto unos como otros tienen acceso a infinidad de recursos tecnológicos que les atraen y los encuentran útiles para realizar tantas tareas como su creatividad despierten. La información y la comunicación fluyen de manera veloz. Y, por lo tanto, los retos a los que se enfrentan estas generaciones, ya que en las escuelas confluyen diferentes generaciones que deben aprender a trabajar de manera colegiada, requieren que las formas en las que se interactúa sean respetuosas y observadoras de lo que cada quien puede aportar. Por lo regular son las otras generaciones las temerosas ante la personalidad tan fuerte que caracteriza a la generación Y. Sin embargo, es necesario que se asuman criterios de apoyo entre unos y otros.

\section{Referencias}

Aldape, T. (2008). Desarrollo de Las Competencias del Docente. Demanda de la Aldea Global. México: Siglo XXI. Editorial Libros en red.

Barrios Rubio, A. (2009). Los jóvenes y la red: usos y consumos de los nuevos medios en la sociedad de la información y la comunicación. Signo y Pensamiento, 28 (Enero-Junio): Recuperado de: http://www.redalyc.org/articulo.oa?id=86011409017

González R. (2015). Manual de Emergencia para Agentes de Cambio Educativo. México: Granica.

Gardner, H. (2011). La inteligencia reformulada. Las inteligencias múltiples en el siglo XXI. Barcelona: Paidós.

Pintado T. y Sánchez, J. (2014). Nuevas Tendencias en Comunicación Estratégica. Madrid: ESIC.

Vázquez, E. y Sevillano M. L. (2011). Dispositivos Digitales Móviles en Educación: El Aprendizaje Ubicuo. Madrid: Narcea. 